\title{
The Development and Application of Automated External Defibrillator
}

\author{
HUANG Yu-rong, LV Hua-qiao, HOU Yuan-yuan \\ Beijing Municipal Institute of Science and Technology Information, Beijing, China \\ hyrta@126.com
}

\begin{abstract}
Using Automated External Defibrillator (AED) to treat sudden cardiac death patients is recognized to be one of the most effective methods at present, and AED has been used widely in the United States, Japan, Britain and other countries, and some countries also had legislated to promote the installation of AED in public places. The development and application of AED in China later many years than in developed countries. This article mainly describes the development and application of AED in developed countries, which provide us reference to promote the development and application of AED in China.

Index Terms - AED, sudden cardiac death, defibrillate
\end{abstract}

\section{Introduction}

Sudden death is one of the biggest challenges the medical profession facing this century, research data reveals that $75 \%$ of the sudden death patients are sudden cardiac death, [1] which is more acute onset, so it is very difficult to treat, and often result in the patient's sudden death, sudden cardiac death is a malignant disease threaten the health and lives of people. The survey data shows that the number people die from sudden cardiac death is 550,000 every year in China, and the proportion of young sudden death is increasing. More than $90 \%$ of the sudden cardiac death patients are ventricular fibrillation, if defibrillation is delayed one minute, the treatment rate will decrease by $7 \%$ to $10 \%$ [2], so early defibrillation is extremely crucial to the survival of sudden cardiac death patients. American Heart Association(AHA) has identified AED is the most effective method to treat sudden cardiac death patients at early time, and has been accepted by the public, and widely used in the United States, Japan and other countries.

AED is designed to treat the cardiac arrest patient, and can be used easily by anyone, even if those never received medical training. Compared with the traditional emergency medical equipment, AED shows specific functionality and efficiency, it can quickly diagnose the patient's heart rhythm, once diagnose with ventricular fibrillation, it will provide voice and demonstration screen guidance, and automatically deliver a shock to defibrillate.

\section{The Development and Application of AED in Foreign Countries}

\section{A. The Development Status}

In the 1970s, Poland emergency physician Stanley Welborn, surgeon Diack, and electrical engineer Rullman firstly invented the automated external defibrillator. [3] In 1979, Medtronic Physio-Control corp. developed the first commercial cardiac defibrillator, which is a semi-automatic defibrillator can identify persistent arrhythmia, and charge and display signal automatically, but operator had to press the "shock" button to defibrillate.[3]The successful development of the Cardiac defibrillator had greatly expanded the concept of outside hospital rescue. Subsequently, many scholars began to develop AED from a wide range, and had confirmed it is sensitive and effective. In the 1990s, Researchers Mattioni and others in the Arizona Heart Institute developed an AED, which made the treatment of cardiac arrest patients more fast and effective, and non-professionals can also use easily. Subsequently, the research and development of AED has been developing in developed countries rapidly, and utilized popularly.

\section{B. The Application}

With the rapid development, AED had become to be one of the main methods that first-aider used to treat sudden cardiac arrest patients in the 1980s. In the 1990s, AED had become to be the basic method to treat sudden cardiac arrest patient. Today, AED is almost as popular as the fire hydrant in some developed countries, and installed in police cars, fire engines, conference centers, government offices, schools, hotels, restaurants, factories, airports, railway stations, trains, subways, and so on.

\section{1) The Application in US}

The United States began to use AED technology in hospital as early as in 1979. In 1990, American Heart Association (AHA) proposed to use AED in public, and formed "AHA Early Defibrillation Project Team", and held twice "Public Access Defibrillation (PAD)" meetings in 1994 and 1997 separately, and proposed "AED is the most promising method to cardiac defibrillation" and "Install AED and hold training course in community", and so on. Under the proposal of AHA and other organizations, the United States began carrying out public defibrillation program in 1995 , experts recommended to extend the application of AED to non-medical personnel in1996. AHA had organized 3.5-4 hours AED training courses in more than 3,000 community training centers (CTCs) across the United States, taught nontraditional rescuers, such as police, firefighters, airline staff and the public to grasp the basic skills to use AED. In November 2000, the United States Congress passed a government proposal to install AED in all the Federal Parliament buildings and civil aviation airports, and required to popularize the application of AED to the public quickly. US 
regulations provided that public places accommodate more than one million people should be equipped with AED. By 2004, every state had finished the AED equipment and legislation, and realized the social coverage of AED in public places across the United States, in some public places, the survival rate of sudden cardiac death rise up to 74\%.[4]

\section{2) The Application in Japan}

In the early time in Japan, the emergency personnel had to use AED to treat sudden cardiac death patients under the guidance of physicians, and only when obtain the consent of shock, they can carry out first aid, and AED is very insufficient at that time, so the proportion of successful treatment had never increased, until July 2004, the Japanese government legislated "non-medical staff to use AED", and allow ordinary people to use AED to treat heart disease patients, meanwhile, Japanese issued 《Non-medical Personnel AED Application Guide》, provides guidance to the general public to utilize AED effectively.

AED is first installed in sports, government agencies, airports and other public places in Japan, and has been installed widely in the stations, movie theaters, gas stations, financial institutions, and even vending machines, with successful treatment events became more and more, all of the middle and primary schools and up to $90 \%$ of colleges and universities had also equipped with AED, the number of AED installed in schools has exceeded 2 million, and more than 33 million units in Japan. In 2008, the well-known real estate developer Sumitomo Realty \& Development equipped with AED in 110 buildings, and also held Lectures about AED application and emergency method to 800 building administrators, Security Guards and front desk staff.

In Japan, in addition to the energetic efforts of the government, enterprises' full cooperation, the non-profit organizations' mobilization, and the public's respect for the value of life, all of these had pushed forward the development and application of AED.

\section{3) The Application in other Countries and Organizations}

In 1999 , British Government enacted policy, which required to install AED in large shopping malls, airports, train stations, entertainment, and implemented public defibrillation program. British Heart Association actively supports the program and provided a large number of AED to hold public defibrillation training courses. International Liaison Committee On Resuscitation (ILCOR) and European Resuscitation Council (ERC) attached great importance to the public defibrillation, and came up with the importance of early defibrillation in the proposal reports in 1997 and 1998 separately, and supported to install AED in the community public places and carry out the AED application training courses, and develop early defibrillation program in the community. After 2000, ILCOR actively advocated to implement the public defibrillation program.

\section{The Development and Application of AED in China}

The development and research of AED in China is later
20 years than developed countries, The First Affiliated Hospital of Soochow University and Fudan University developed the first homebred AED at the end of 2006, it is the first independent intellectual property rights AED, which has been used to complete more than 20 cases of animal experiments, and the research team applied for nine related patents.[5]In 2007, after the research and development for nearly four years, Beijing M\&B Electronic Instruments Co., Ltd successfully developed the only low-energy, high success rate and low myocardial injury characteristic biphasic AED, the prototype safe and all of the performance indicators reached the international standard, and was completed the European Union CE certification in December 2009.

In 2006, Beijing Capital International Airport installed eleven AEDs at the first terminal, marking the AED program was officially launched in our country. In order to enhance cardiac arrest survival rate during the Olympic Games, Beijing municipal government purchased 400 AEDs in 2008, and 300 AEDs were separately installed in emergency ambulances, Olympic venues, the rest were installed at Peking University People's Hospital, The Second Affiliated Hospital of Harbin Medical University, Shanghai Chest Hospital Affiliated to Shanghai Jiaotong University and First Affiliated Hospital of Kunming Medical University, meanwhile, Beijing municipal government held training courses for security and medical personnel. Before the opening of the World Expo 2010 in Shanghai, the Expo area, airports and other public places were equipped with AEDs, and held training courses for relevant personnel.

Chinese Heart Rhythm Society (CHRS) and Chinese Medical Association (CMA) had played very important role in promoting the implementation of AED program. In January 2008, CHRS issued initiatives "Reduce Sudden Death, and Advance AED Process" in Harbin academic annual meeting, and held the Opening ceremony for the AED Training Center of the Second Affiliated Hospital of Harbin Medical University. Subsequently, AED Training Base also been set up in Shanghai, Kunming, Xinjiang and other places, which was very important to improve the survival rate of sudden cardiac death patients outside hospital in the future, the development and application of AED has entered the promotion and popularization stage in China.

\section{The Conclusion}

The successful application of AED in foreign countries not only stems from the firm legislation, but also from the respect for life, their successful experience proved to the world that AED can play the largest role in first aid when it go out of the hospital and is popularized to the society. The development and application of AED later for many years in China than in foreign countries, and only a handful of research institute developed the AED development, even if some research institute have developed independent intellectual property rights $\mathrm{AED}$, but it is extremely difficult to industrialized in our country due to the lack of pay attention to, there is still a long way to go. 
Summarize the successful experience of the AED popularization and application in developed countries, there are three problems to be solved in China:

1) To strengthen the technology research and development of AED, develop AED with independent intellectual property, and solve the problem that can't be popularized due to the high prices of imported AED.

2) To strengthen the popularization and training of AED among the public, and to enhance the public awareness of AED treatment and application level, so that ordinary people can manipulate the AED easily, improve the efficiency of treatment out of the hospital.

3) To strengthen legislation and supervision. Government need to pass legislation to promote the popularization and application of AED, and stipulate that the equipment of a sufficient number of AED in various public places, such as large shopping malls, gym, entertainment, schools, airports, railway stations, community, large factory, and so on, and Non-medical personnel can use AED to treat sudden cardiac death patients, and hold training course to relevant staff.
Once the government and people pay more attention to the development and application of AED, and promote the popularization quickly, then thousands of sudden cardiac death patients in China can be treated timely and effectively, and the health of our people will be greatly improved.

\section{References}

[1]. Zhao Da-ming. "Automated External Defibrillator and "public start defibrillation' program." Journal of Clinical Emergency, vol.9, no.6, pp.365-366, 2008.

[2]. Jiang Jian, YU Jin-de. "Modern emergency internal medicine," Science Press, pp.38-39, 1999.

[3]. Stoddard FG, Cummins RO. "The forgotten first chapterin AED history: modern computerized devices have origin in Oregon trio's 1970's invention." Curr Emerg Cardiac Care, no.8, pp.1-4, 1997.

[4]. Valenzuela TD, Roe DJ, Nichol G, Clark LL, Spaite DW, Hardman RG. "Outcomes of rapid defibrillation by security officers after cardiac arrest in casinos." N Engl J Med. ' vol.343, no.17, pp.1206-1209, 2000.

[5]. GuWei, Li Chun-sheng. "Automated External Defibrillator: creating a new era human conquest sudden death," China Medical Device Information, 2010, vol.16, no.9, pp.1-3, 2010. 